\title{
Violência contra o idoso durante a pandemia COVID-19: revisão de escopo
}

\author{
Elder abuse during the COVID-19 pandemic: a scoping review
}

Violencia contra adultos mayores durante la pandemia de COVID-19: revisión de alcance

\author{
Ana Maria Ribeiro dos Santos ${ }^{1}$ i nttps://orcid.org/0000-0002-5825-5335 \\ Guilherme Guarino de Moura Sá2 ${ }^{10}$ https://orcid.org/0000-0003-3283-2656 \\ Ana Angélica Oliveira de Brito ${ }^{1}$ ic https:///orcid.org/0000-0001-866-4729 \\ Julyanne dos Santos Nolêto ${ }^{1}$ ic https://orcid.org/0000-0002-0342-6838 \\ Rouslanny Kelly Cipriano de Oliveira ${ }^{1}$ if https://orcid.org/0000-0002-4843-6079
}

Santos AM, Sá GG, Brito Oliveira RK. Elder abuse during the COVID-19 pandemic: a scoping review. Acta Paul Enferm. 2021;34:AAPE000336.

DOI

http://dx.doi.org/10.37689/actaape/2021AR00336

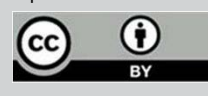

Idoso; Violência; Abuso de idosos; COVID-19;

Pandemias

Keywords

Aged; Violence; Elder abuse; COVID-19; Pandemics

Descriptores

Anciano; Violencia; Abuso de ancianos; COVID-19;

Pandemia

Submetido 2 de Fevereiro de 2021 Aceito 26 de Julho de 2021

Autor correspondente Guilherme Guarino de Moura Sá E-mail: guilherme_mourasa@hotmail.com

\section{Resumo}

Objetivo: Mapear as evidências científicas disponíveis acerca da violência contra o idoso durante a pandemia COVID-19.

Métodos: Revisão de escopo que teve como questão de pesquisa "Quais são as evidências científicas disponíveis acerca da violência contra o idoso durante a pandemia COVID-19?". A seleção dos estudos ocorreu em novembro de 2020, a partir de buscas nas bases PubMed, EMBASE, CINAHL, Web of Science, Scopus e LILACS, consulta ao Google Scholar e busca manual nas listas de referências dos estudos que fizeram parte da amostra. Incluíram-se estudos publicados de 2019 a novembro de 2020 e não houve restrição de idioma. A análise ocorreu pelo método de redução de dados.

Resultados: Foram incluídos 12 artigos, quatro (33,3\%) eram estudos reflexivos, dois (16,7\%) de opinião, duas $(16,7 \%)$ cartas ao editor, duas $(16,7 \%)$ revisões narrativas, uma $(8,3 \%)$ nota breve e um $(8,3 \%)$ editorial. Os estudos foram sintetizados em seis categorias sobre a violência contra 0 idoso durante a pandemia COVID-19: 1) riscos para violência contra 0 idoso na comunidade; 2) riscos para violência contra 0 idoso institucionalizado; 3) condições que podem agravar os riscos; 4) ações políticas e organizacionais para enfrentamento da violência; 5) ações nas ILPI para enfrentamento da violência; 6) ações profissionais e sociais para enfrentamento da violência.

Conclusão: As evidências disponíveis na literatura são de estudos teóricos, que discutiram os riscos para violência contra o idoso na comunidade e institucionalizado, condições de agravamento dos riscos e ações para enfrentamento. Existe lacuna nas evidências de estudos empíricos sobre 0 tema.

\section{Abstract}

Objective: To map the available scientific evidence about elder abuse during the COVID-19 pandemic.

Methods: This is a scoping review whose research question was "What is the scientific evidence available about elder abuse during the COVID-19 pandemic?". The selection of studies took place in November 2020, based on searches in the PubMed, EMBASE, CINAHL, Web of Science, Scopus and LILACS databases, Google Scholar consultation and manual search in the reference lists of studies that were part of the sample. Studies published from 2019 to November 2020 were included and there was no language restriction. Analysis took place using the data reduction method.

Results: Twelve articles were included: four (33.3\%) reflective studies, two (16.7\%) opinion, two (16.7\%) letters to the editor, two (16.7\%) narrative reviews, one (8.3\%) brief note and one (8.3\%) editorial. The studies were summarized into six categories of elder abuse during the COVID-19 pandemic: 1) risks for elder abuse 
in the community; 2) risks for institutionalized elder abuse; 3) conditions that can aggravate the risks; 4) political and organizational actions to fight violence; 5) actions in ILPI to fight violence; 6) professional and social actions to cope with violence.

Conclusion: The evidence available in the literature is from theoretical studies, which discussed the risks of elder abuse in the community and institutionalized, conditions of aggravation of risks and actions for coping. There is a gap in the evidence from empirical studies on the subject.

\section{Resumen}

Objetivo: Mapear las evidencias científicas disponibles acerca de la violencia contra los adultos mayores durante la pandemia de COVID-19.

Métodos: Revisión de alcance con la siguiente pregunta de investigación: “¿Cuáles son las evidencias científicas disponibles acerca de la violencia contra los adultos mayores durante la pandemia de COVID-19?". La selección de los estudios se llevó a cabo en noviembre de 2020, a partir de búsquedas en las bases PubMed, EMBASE, CINAHL, Web of Science, Scopus y LILACS, consulta en Google Scholar y búsqueda manual en las listas de referencias de los estudios que formaron parte de la muestra. Se incluyeron estudios publicados de 2019 a noviembre de 2020 y no hubo restricción de idioma. El análisis se realizó con el método de reducción de datos.

Resultados: Se incluyeron 12 artículos, de los cuales cuatro (33,3 \%) eran estudios reflexivos, dos (16,7 \%) de opinión, dos (16,7 \%) cartas al editor, dos $(16,7 \%)$ revisiones narrativas, una (8,3 \%) nota breve y un (8,3\%) editorial. Los estudios fueron sintetizados en seis categorías sobre la violencia contra los adultos mayores durante la pandemia de COVID-19: 1) riesgos de violencia contra los adultos mayores en la comunidad; 2) riesgos de violencia contra Ios adultos mayores institucionalizados; 3) condiciones que pueden agravar los riesgos; 4) acciones políticas y organizacionales para el enfrentamiento a la violencia; 5) acciones para el enfrentamiento a la violencia en las ILPI (instituciones de larga permanencia para adultos mayores); y 6) acciones profesionales y sociales para el enfrentamiento a la violencia.

Conclusión: Las evidencias disponibles en la literatura son de estudios teóricos en los que se discuten los riesgos de violencia contra los adultos mayores institucionalizados y en la comunidad, las condiciones de agravamiento de los riesgos y las acciones para el enfrentamiento. Existe un vacío en las evidencias de estudios empíricos sobre el tema.

\section{Introdução}

O mundo acompanha a evolução da COVID-19 desde 9 de janeiro de 2020, quando autoridades chinesas relataram um surto em Wuhan, na República Popular da China, de um novo tipo de coronavírus humano, o SARS-CoV-2.$^{(1)}$ Devido a rápida disseminaçáo da doença para outros países, a Organização Mundial da Saúde (OMS) declarou-a Pandemia em 11 de março de 2020. ${ }^{(2)}$ A partir de entáo, os governos passaram a recomendar medidas severas para controle de sua disseminação, em decorrência da ausência de evidências, de droga ou vacina aprovados para tratamento e prevenção. ${ }^{(3)}$

Globalmente, até 7 de março de 2021, quase 120 milhóes de casos e mais de dois milhóes e meio de mortes foram relatados, destacando-se que recentemente, o aumento da incidência de casos tem sido mais relevante no continente Europeu. Na Região das Américas, os Estados Unidos e Brasil são os países que tem apresentado maior número de novos casos, com um cumulativo de mortes de 519.075 e 262.770 , respectivamente. ${ }^{(4)}$

Ao longo do período pandêmico, os idosos estão entre as pessoas mais afetadas pela doença. Em relação a mortalidade, identificou-se risco de 3,6\% na faixa dos 60 anos, com aumento para $8,0 \%$ e $14,8 \%$ na faixa de 70 e 80 anos e mais, respectivamente. ${ }^{(5)}$ Na mesma perspectiva, estudo chinês evidenciou que pessoas com mais de 55 anos tiveram mortalidade três vezes maior, provavelmente relacionadas as comorbidades pré-existentes. ${ }^{(6)}$

Nesse sentido, para conter a transmissão e reduzir a disseminação da COVID-19 se tem recomendado o reforço de procedimentos preventivos náo farmacológicos, dentre eles o distanciamento social ou espacial, fato observado tanto no ambiente familiar quanto nos serviços de atendimento a idosos. No entanto, apesar da necessidade desse procedimento, pode-se observar consequências a curto e longo prazo na saúde da população idosa, com destaque para a ocorrência de violência. ${ }^{(7)}$

A violência é representada por açóes ou omissóes cometidas uma ou muitas vezes, que prejudicam a integridade física e emocional e impedem o desempenho do papel social do idoso, uma vez que pode atingir a preservação da sua imagem, identidade e autonomia, assim como abarcar seus valores, ideias e crenças, até seus espaços e objetos pessoais. Portanto, trata-se de evento que pode se manifestar de várias formas: abuso físico, psicológico, sexual, abandono, negligência, abusos financeiros e autonegligência. ${ }^{(8)}$

Esse problema não é um fenômeno novo, acontece com até $10 \%$ dos idosos a cada ano e as vítimas 
podem sofrer mais de um tipo de violência simultaneamente. ${ }^{(9)}$ No entanto, é possível constatar que o isolamento social durante a pandemia exacerbou fatores que colocam os idosos em maior perigo, tanto em relaçáo aos riscos ligados ao perpetrador da violência quanto aqueles relacionados a vulnerabilidade do idoso vitimado, tais como: problemas com saúde física e mental; uso de substâncias; dependência; problemas com estresse e enfrentamento; atitudes, relacionamento e vitimização. ${ }^{(10)}$

A OMS relata aumento de cerca de dez vezes de abusos e negligências contra as pessoas mais velhas durante a pandemia e alerta sobre o maior risco de violência entre aqueles idosos com problemas de mobilidade, que enfrentam barreiras para acessar informaçóes confiáveis usando tecnologias, que apresentam dependência física e também os mais vulneráveis a infecçóes. ${ }^{(11)}$

No Brasil, as denúncias de violência contra as pessoas idosas, registradas pelo "Disque 100", no período de março a maio de 2020, passaram de 3 mil em março para 8 mil em abril e 17 mil em maio, correspondendo a aumento de $267 \%$ e $567 \%$, no período. ${ }^{(12)}$ Entretanto, a temática ainda permanece pouco estudada no país em relação a esse fato específico, pois, em ampla busca na literatura científica internacional, constatou-se incipiência de pesquisas publicadas até o momento, que apresentassem riscos e açóes de enfrentamento a violência contra a pessoa idosa durante o período pandêmico. Nesse sentido, sua investigaçáo poderia possibilitar a identificação de estratégias de açáo para auxiliar cuidadores formais e informais, profissionais de saúde e gestores em relação a prevenção de violência contra idosos, minimizando sua ocorrência.

Este estudo objetivou mapear as evidências científicas disponíveis acerca da violência contra o idoso durante a pandemia COVID-19.

\section{Métodos}

Trata-se de revisão de escopo conduzida conforme recomendaçóes do Instituto Joanna Briggs (JBI), e seguiu as fases: definição do objetivo e questão de pesquisa; definição dos critérios de inclusão; plane- jamento da estratégia de busca; busca de estudos; seleção; extração dos dados; análise dos dados; apresentação dos resultados; sumarização das evidências mapeadas em relaçáo ao objetivo da revisão. ${ }^{(13,14)}$

Inicialmente, constatou-se, a partir de busca no site do JBI, a inexistência de revisóes que propuseram investigar a temática. As fases da revisão foram previamente estruturadas em protocolo elaborado pelos pesquisadores. A questão de pesquisa foi: Quais são as evidências científicas disponíveis acerca da violência contra o idoso durante a pandemia COVID-19? Essa questáo incorporou os elementos do mnemônico PCC (População, Conceito e Contexto), de forma que a população definida foi de idosos, o conceito de interesse foi a violência e o contexto investigado foi a pandemia COVID-19.

Os critérios de inclusão foram: estudos com os mais variados delineamentos metodológicos, que abordassem qualquer tipo de violência contra o idoso durante a pandemia COVID-19; sem restrição de idioma; publicados entre 2019 até 30 de novembro de 2020. Justifica-se esta delimitação temporal, uma vez que o coronavírus humano SARS-CoV-2 foi identificado pela primeira vez no ano de 2019 . (1) Foram excluídos estudos que não possuíam informaçóes pertinentes para a população, conceito e contexto de interesse.

A estratégia de busca foi planejada em três etapas distintas. $\mathrm{Na}$ primeira, realizou-se levantamento em seis bases de dados: National Center for Biotechnology Information (NCBI/PubMed); Excerpta Medica Database (EMBASE); Cumulative Index to Nursing and Allied Healh Literature (CINAHL) via coleção principal Thomson Reuters; Web of Science via coleção principal (Clarivate Analytics); Scopus (Elsevier); e Latin American and Caribbean Health Sciences Literature (LILACS) via Biblioteca Virtual em Saúde (BVS). Na segunda etapa, foi empregada pesquisa de literatura cinzenta, por meio de consulta ao Google Scholar. A terceira etapa consistiu em busca manual na lista de referências dos estudos incluídos nas etapas anteriores, com vistas rastrear estudos adicionais náo identificados nas bases de dados.

A busca dos estudos nas bases de dados ocorreu em 30 de novembro de 2020, a partir do portal de periódicos da Coordenação de Aperfeiçoamento 
de Pessoal de Nível Superior (CAPES), por meio de acesso remoto da Comunidade Acadêmica Federada (CAFe) e registro na Universidade Federal do Piauí. Para busca dos estudos foram selecionados descritores controlados do Medical Subject Headings (MeSH), Embase Subject Headings (EMTREE), CINAHL Headings e Descritores em Ciências da Saúde (DeCS), de forma a contemplar os elementos do mnemônico PCC: idosos, violência e pandemia COVID-19. Adicionalmente, foram selecionados descritores não controlados, estabelecidos a partir dos sinônimos dos descritores controlados das respectivas bases.

Para condução da busca de alta sensibilidade nas bases de dados, os descritores de cada conjunto da estratégia PCC foram combinados entre si com o conector booleano $\mathrm{OR}$, para obtenção de combinação aditiva. Em seguida, obteve-se combinação restritiva a partir do cruzamento, entre si, de cada conjunto da estratégia PCC com o conector AND. A estratégia de busca respeitou as peculiaridades de cada base e não foram adicionados limitadores de idioma.

O quadro 1 apresenta a expressão de busca na PubMed. Expressóes de busca semelhantes foram obtidas em cada base de dados.

Quadro 1. Expressão de busca na base de dados PubMed

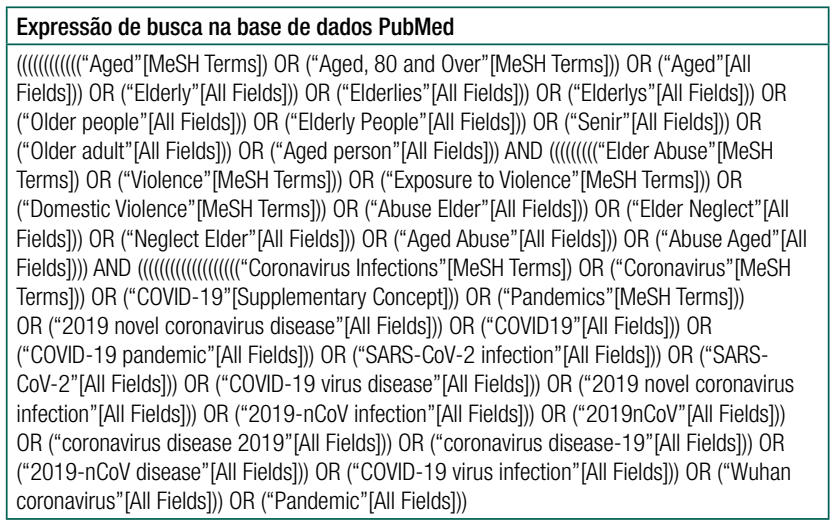

Para auxiliar no armazenamento, organização, identificação dos estudos duplicados e seleção dos artigos, as referências identificadas foram importadas no aplicativo Rayyan, desenvolvido pelo Qatar Computing Research Institute (QCRI). ${ }^{(15)}$ A seleção dos estudos foi conduzida por três revisores independentes. Os resultados obtidos foram compara- dos e observou-se divergência entre os revisores em $15,7 \%$ das decisóes. Nesse caso, um quarto revisor avaliou os artigos incluídos e excluídos por cada revisor e tomou a decisão final.

Para extração e mapeamento dos dados dos estudos incluídos utilizou-se instrumento adaptado de formulário recomendado pelo JBI. ${ }^{(16)}$ Foram extraídas as informaçóes: ano; país de origem dos autores; título do estudo; periódico; tipo de estudo; principais evidências acerca da violência contra o idoso durante a pandemia COVID-19.

Para sumarizar as informaçóes e apresentar lacunas identificadas, utilizou-se o método de redução dos dados, por meio de leitura crítica e classificação dos resultados em categorias conceituais. (17) $\mathrm{O}$ mapeamento dos resultados ocorreu na forma descritiva, com apresentação de contagem simples e sumarização em quadros. Esse processo foi realizado separadamente por duas equipes com dois revisores, cada, e, na sequência, avaliado pela pesquisadora principal. $\mathrm{O}$ relato desta revisão seguiu a lista de verificação do Preferred Reporting Items for Systematic reviews and Meta-Analyses extension for Scoping Reviews (PRISMA-ScR). ${ }^{(18)}$

\section{Resultados}

As estratégias de busca permitiram identificar 478 estudos, sendo 374 na Scopus, 50 na EMBASE, 23 na PubMed, 22 na Web of Science, oito na CINAHL e um na LILACS. Após exclusão dos estudos duplicados e aplicação dos critérios de elegibilidade, foram selecionados dez estudos. Adicionalmente, foram identificados dois estudos, após consulta ao Google Scholar. $\mathrm{Na}$ busca manual nas listas de referências dos estudos incluídos náo foram encontrados novos estudos que respondessem à questão de pesquisa. Assim, 12 estudos compuseram a amostra final. O fluxo do processo de seleção dos estudos é ilustrado na figura 1.

Todos os estudos incluídos nesta revisão foram publicados no ano de 2020, em periódicos de áreas distintas, tais como gerontologia, geriatria, enfermagem, serviço social, emergência, psiquiatria e saúde coletiva. Em relação ao idioma, dez $(83,4 \%)$ artigos foram escritos em inglês, um $(8,3 \%)$ em francês e um 


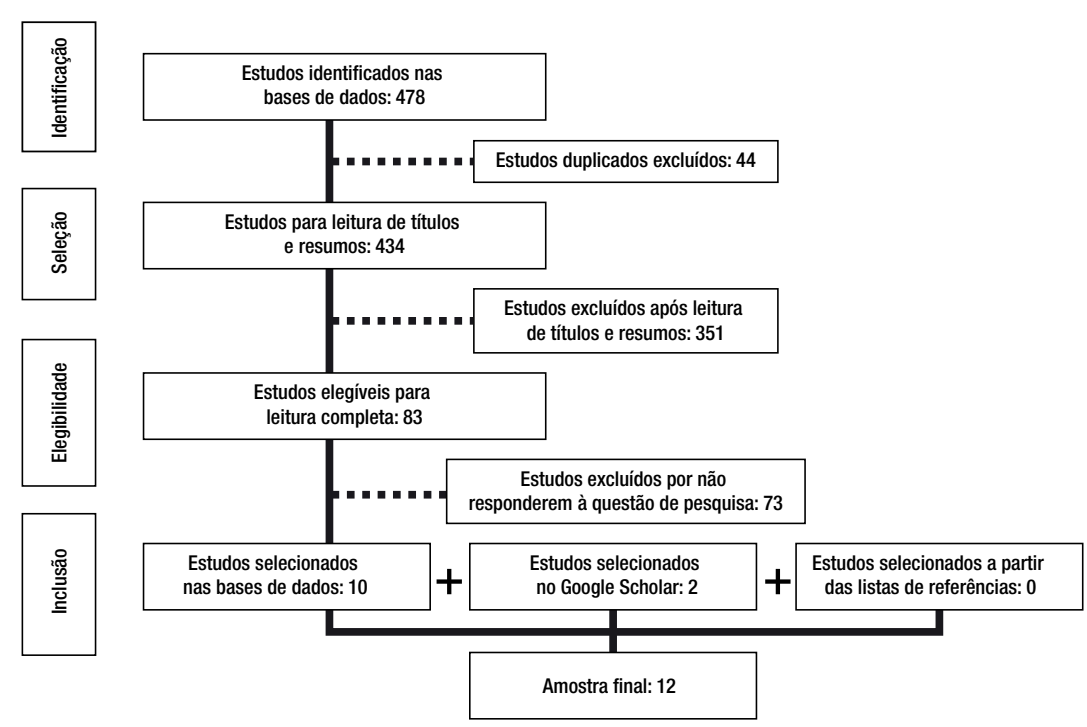

Figura 1. Fluxograma do processo de seleção dos estudos, elaborado a partir das recomendações do PRISMA-ScR

$(8,3 \%)$ em português. No tocante ao tipo de estudo, observou-se que não foram identificadas pesquisas empíricas que respondessem à questão de pesquisa desta revisão, uma vez que foram selecionados quatro $(33,3 \%)$ estudos reflexivos, dois $(16,7 \%)$ de opinião, duas $(16,7 \%)$ cartas ao editor, duas $(16,7 \%)$ revisóes narrativas, uma $(8,3 \%)$ nota breve e um $(8,3 \%)$ editorial. As características dos estudos incluídos encontram-se detalhadas no quadro 2.

De forma geral, os estudos analisados refletiram questóes que permeiam a violência em diferentes cenários, tais como o domicílio e instituiçôes de longa permanência, e não abordaram tipos de violência específicos. Além disso, buscaram contemplar fatores sociais, institucionais e políticos que permeiam a pandemia.
Nesse sentido, ao considerar as diferentes abordagens sobre o tema, as evidências científicas foram agrupadas em seis categorias: 1) riscos para violência contra o idoso na comunidade durante a pandemia COVID-19;(19,23,24,26,27,29) 2) riscos para violência contra o idoso institucionalizado durante a pandemia COVID-19;(21,22) 3) condiçôes que podem agravar os riscos para violência contra $o$ idoso durante a pandemia COVID-19;(19,24,28,29) 4 ) açóes políticas e organizacionais para enfrentamento da violência contra o idoso durante a pandemia COVID-19;(19,20,25,30) 5) açôes nas Instituiçôes de Longa Permanência para Idosos (ILPI) para enfrentamento da violência contra o idoso durante a

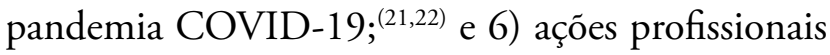

Quadro 2. Estudos incluídos na revisão de escopo, segundo título, periódico, tipo de estudo e país de origem

\begin{tabular}{|c|c|c|c|}
\hline Título do artigo & Periódico & Tipo de estudo & País de origem \\
\hline $\begin{array}{l}\text { Effects of the COVID-19 outbreak on elder mistreatment and response in New York City: initial } \\
\text { lessons }^{(19)}\end{array}$ & Journal of Applied Gerontology & Reflexivo & Estados Unidos \\
\hline Protecting the elderly through and beyond the Covid-19 lockdown ${ }^{(20)}$ & Journal of Community Nursing & Opinião & Reino Unido \\
\hline The coronavirus and the risks to the elderly in long-term care ${ }^{(21)}$ & Journal of Aging \& Social Policy & Reflexivo & Canadá \\
\hline Statutory neglect and care in a pandemic ${ }^{(22)}$ & International Social Work & Nota breve & Reino Unido \\
\hline Strengthening our intuition about elder abuse $e^{(23)}$ & Annals of Emergency Medicine & Editorial & Estados Unidos \\
\hline Elder abuse in the time of COVID-19 - increased risks for older adults and their caregivers ${ }^{(24)}$ & The American Journal of Geriatric Psychiatry & Opinião & Estados Unidos \\
\hline Elder abuse in the COVID-19 era(25) & Journal of the American Geriatrics Society & Carta ao editor & Estados Unidos \\
\hline Psychological abuse in the elderly during exchanging news of COVID-19(26) & Iranian Journal of Psychiatry and Behavioral Sciences & Carta ao editor & Iran \\
\hline $\begin{array}{l}\text { Suicidal behavior in light of COVID-19 outbreak: clinical challenges and treatment } \\
\text { perspectives }^{(27)}\end{array}$ & L'Encéphale & Revisão narrativa & França \\
\hline $\begin{array}{l}\text { Impact of COVID-19 pandemic restrictions on community-dwelling caregivers and persons } \\
\text { with dementia }{ }^{(28)}\end{array}$ & $\begin{array}{l}\text { Psychological Trauma: Theory, Research, Practice, and } \\
\text { Policy }\end{array}$ & Reflexivo & Estados Unidos \\
\hline Impact of COVID-19 pandemic and lockdown on elder abuse ${ }^{(29)}$ & Journal of Geriatric Care and Research & Revisão narrativa & Índia \\
\hline $\begin{array}{l}\text { Violência contra idosos durante a pandemia de Covid-19 no Brasil: contribuições para seu } \\
\text { enfrentamento }{ }^{(30)}\end{array}$ & Ciência \& Saúde Coletiva & Reflexivo & Brasil \\
\hline
\end{tabular}


Quadro 3. Síntese das evidências sobre violência contra o idoso durante a pandemia COVID-19

\begin{tabular}{|c|c|}
\hline \multicolumn{2}{|c|}{ Riscos para violência contra o idoso na comunidade durante a pandemia COVID-19 } \\
\hline $\begin{array}{l}\text { - Isolamento social. } \\
\text { - Solidão. } \\
\text { - Dependência funcional. } \\
\text { - Deficiência. } \\
\text { - Doença física ou mental. } \\
\text { - Perda/diminuição de renda. } \\
\text { - Dependência financeira. } \\
\text { - Idoso com baixa escolaridade. } \\
\text { - Moradia em local de difícil acesso. }\end{array}$ & $\begin{array}{l}\text { - Comprometimento cognitivo. } \\
\text { - Acesso reduzido aos recursos da comunidade. } \\
\text { - Cancelamentos de visitas presenciais. } \\
\text { - Aumento do consumo de substâncias. } \\
\text { - Inaptidão do idoso em manipular tecnologias para gestão dos cuidados de saúde remotos. } \\
\text { - Conversas/palavras que podem causar medo no idoso, como por exemplo "a doença só mata } \\
\text { os idosos". } \\
\text { - Evento de vida estressante, como perda do cônjuge. }\end{array}$ \\
\hline \multicolumn{2}{|l|}{ Riscos para violência contra o idoso institucionalizado durante a pandemia COVID-19 } \\
\hline $\begin{array}{l}\text { - Diminuição ou proibição de visitas familiares em Instituições de Longa Permanência para Idosos } \\
\text { (LLPI). } \\
\text { - Decisões políticas que desconsideram as ILPI. }\end{array}$ & $\begin{array}{l}\text { - Estabelecimento degradado, superlotado ou insuficiente. } \\
\text { - Alta rotatividade de profissionais. }\end{array}$ \\
\hline \multicolumn{2}{|l|}{$\begin{array}{l}\text { Condições que podem agravar os riscos para violência contra o idoso durante a pandemia } \\
\qquad \text { COVID-19 }\end{array}$} \\
\hline $\begin{array}{l}\text { - Dificuldades de acesso a cuidados e suprimentos necessários. } \\
\text { - Intenção do cuidador em substituir o contato pessoal por contato por meio de tecnologias } \\
\text { virtuais. } \\
\text { - Descontinuidade do cuidado por cuidadores formais ou equipe multidisciplinar de saúde } \\
\text { domiciliar. } \\
\text { - Dificuldade na detecção de abuso, pela incapacidade de reconhecer se o idoso está sozinho no } \\
\text { momento de avaliação, por meio de visita virtual. } \\
\text { - Aumento da interação no ambiente domiciliar, que pode agravar problemas interpessoais } \\
\text { existentes. }\end{array}$ & $\begin{array}{l}\text { - Membros da família desempregados/instabilidade financeira, que podem apropriar-se das } \\
\text { economias, rendimentos e auxilios financeiros do idoso. } \\
\text { - Idoso que não possui telefone celular ou não utiliza por capacidade limitada de comunicação, } \\
\text { devido fatores connitivos ou deficiências físicas. } \\
\text { - Aumento da ansiedade dos cuidadores, devido estressores financeiros, demandas de tempo e } \\
\text { preocupações com a saúde. } \\
\text { - Aumento da sobrecarga de cuidadores de idosos com demência, problemas comportamentais } \\
\text { ou depressão. }\end{array}$ \\
\hline \multicolumn{2}{|c|}{ Ações políticas e organizacionais para enfrentamento da violência contra o idoso durante a pandemia COVID-19 } \\
\hline \multicolumn{2}{|c|}{$\begin{array}{l}\text { - Criar políticas voltadas ao apoio econômico das famílias de baixa renda. } \\
\text { - Criar abrigos de emergência especializados. } \\
\text { - Conscientizar a sociedade acerca da rede de proteção do idoso. } \\
\text { - Manter e ampliar a atuação e parcerias de delegacias, conselhos, associações do idoso. } \\
\text { - Definir departamento de polícia responsável pela violência doméstica. } \\
\text { - Manter as investigações das denúncias de violência. } \\
\text { - Realizar adaptaçố no sistema judicial, que permita processos remotos durante a pandemia. } \\
\text { - Incentivar e promover reuniões remotas com equipe multidisciplinar de gestão da violência contra idosos. } \\
\text { - Utilizar a telemedicina para avaliar o público idoso. } \\
\text { - Capacitar equipe multidisciplinar para triagem de casos. } \\
\text { - Buscar apoio de organizações religiosas e sem fins lucrativos em programas de prevenção da violência. } \\
\text { - Buscar apoio de vizinhos, porteiros e pessoas da comunidade para identificação de idosos em risco. }\end{array}$} \\
\hline \multicolumn{2}{|c|}{ Ações nas ILPI para enfrentamento da violência contra o idoso durante a pandemia COVID-19 } \\
\hline \multicolumn{2}{|c|}{$\begin{array}{l}\text { - Ampliar as inspeções. } \\
\text { - Certificar que as instalações e o dimensionamento de profissionais podem atender necessidades dos residentes. } \\
\text { - Certificarar a contratação de profissionais substitutos daqueles afastados por adoecimento. } \\
\text { - Avaliar o impacto das legislações sobre negligência durante a pandemia nas ILPI. } \\
\text { - Abordar a negligência estatutária em reuniões de equipe institucional e procedimentos para denúncia em ILPI. } \\
\text { - Realizar campanha e ações políticas em apoio aos idosos de ILPI por meio de associações/conselhos profissionais. }\end{array}$} \\
\hline \multicolumn{2}{|c|}{ Ações profissionais e sociais para enfrentamento da violência contra o idoso durante a pandemia COVID-19 } \\
\hline $\begin{array}{l}\text { - Promover campanhas educativas para o público geral e idosos sobre a violência contra a pessoa } \\
\text { - Incentivar o autocuidado e práticas de bem-estar para cuidadores de idosos, durante o isolament } \\
\text { - Avaliar a capacidade de autocuidado do idoso e cuidados realizados pelos cuidadores, durante co } \\
\text { - Promover estratégias de conscientização da população sobre sinais de violência contra o idoso. } \\
\text { - Incentivar a terapia comportamental e cognitiva para idosos e cuidadores. } \\
\text { - Estimular intervenções artísticas voltadas para a promoção da saúde mental de idosos e cuidador } \\
\text { - Identificar comportamentos suspeitos, como recusar falar ao telefone e aumento do sono durante } \\
\text { - Realizar ligação telefônica para o idoso sem prévio aviso, para que o cuidador não planeje estar p p } \\
\text { - Compartilhar o cuidado ao idoso entre os moradores do domicílio, com vistas reduzir a sobrecarg } \\
\text { - Promover apoio a cuidadores de idosos com sobrecarga. }\end{array}$ & $\begin{array}{l}\text { dosa. } \\
\text { social. } \\
\text { ntato virtual. } \\
\text { es. } \\
\text { o dia. } \\
\text { resente. } \\
\text { dos cuidadores. }\end{array}$ \\
\hline
\end{tabular}

e sociais para enfrentamento da violência contra o idoso durante a pandemia COVID-19. ${ }^{(19,20,24,25,27,30)}$

A síntese das evidências científicas é apresentada no quadro 3.

\section{Discussão}

No contexto da pandemia COVID-19, a vulnerabilidade da pessoa idosa, seja nos aspectos físicos, emo- cionais, cognitivos e financeiros, consistiu em forte indicador de violência contra essa população. $\mathrm{O}$ abuso de idosos resulta da combinaçáo de fatores relacionados aos próprios idosos, cuidadores, outras pessoas do círculo social e contexto no qual ele está inserido. ${ }^{(24,25)}$ Desse modo, as estratégias de prevenção e identificação da violência durante a pandemia tornam-se complexas e devem envolver diferentes atores da sociedade.

$\mathrm{O}$ isolamento social, como estratégia preventiva do contágio pelo vírus SARS-CoV-2, foi acompa- 
nhado de problemas sociais e de solidão dos idosos. Estudos relataram que, a interrupçáo ou redução da oferta de serviços de saúde e de proteção social, como serviços comunitários, contribuíram para a manutenção, agravamento ou surgimento de casos de violência contra a pessoa idosa. ${ }^{(19,23,30)}$ Além disso, essa medida ocasionou aumento da interaçáo entre idosos e seus familiares e cuidadores, e por conseguinte, pode ter agravado questóes interpessoais que resultaram em discussóes e aumento do risco de abuso verbal, físico ou negligência. ${ }^{(29)}$

Diante disso, verifica-se a necessidade de ações de promoção da saúde que considerem as relaçóes interpessoais entre a pessoa idosa, a família e a comunidade, que possam ser motivadores de violência contra a pessoa idosa. Ademais, tais ações também devem ser consideradas na abordagem de problemas de saúde em que o isolamento social seja medida preventiva.

Os idosos com dependência para realização das atividades de vida diária e aqueles com doença demencial apresentam maiores riscos de abuso e negligência. ${ }^{(24,27)}$ Somado a isso, têm-se o fato de que os principais perpetradores da violência geralmente são pessoas próximas ao idoso, principalmente familiares que, além de cuidar do idoso, acumulam outras responsabilidades, tensóes associadas a dificuldades financeiras ou traumas psicológicos devido a pandemia. ${ }^{(19,24,30)}$

Desse modo, a avaliação multidimensional do idoso e da sobrecarga do cuidador torna-se instrumento para os profissionais de saúde identificarem riscos ou sinais de violência e prevenirem a maior sobrecarga de cuidadores. Ademais, o genograma familiar é instrumento que está ao alcance dos profissionais da saúde, e o enfermeiro pode utilizá-lo para facilitar o plano terapêutico na saúde da família, de modo auxiliar no entendimento da composição e dinâmica da família e suas relaçóes.

Cabe destacar que, nesse cenário, as demandas de cuidado às pessoas idosas, sobretudo para o enfermeiro, são ampliadas, uma vez que além de tratar os casos de COVID-19, precisam estar atentos para identificação de riscos e sinais de violência. Nesse sentido, as sobrecargas física e emocional desses profissionais devem ser levadas em conta no di- mensionamento de pessoal e na valorização frente ao cuidado geriátrico. Destarte, urge a implantação de processos de formação e educação continuada a esses profissionais, acerca da identificação de casos de violência contra essa parcela da população, além da abordagem frente a casos suspeitos.

O isolamento social somado ao abuso do consumo de substâncias, tanto por idosos como por cuidadores, pode ampliar os riscos para as diversas formas de violência. ${ }^{(24,29,30)}$ Destaca-se, ainda, que o isolamento pode contribuir para o desenvolvimento de problemas de saúde mental que comprometem o bem-estar dos idosos, tais como insônia, perda de apetite, depressão e ideação suicida. ${ }^{(27,30)}$ Achados semelhantes foram encontrados em estudo com adultos, durante a pandemia $\mathrm{H} 1 \mathrm{~N} 1$, e observou associação entre intolerância e incerteza, autopercepção alterada e ansiedade. ${ }^{(31)}$

Logo, faz-se necessária a implementação de açôes de promoção de saúde mental e física, com recomendação de terapias cognitivas e comportamentais e atividades artísticas, alinhadas aos demais cuidados com o bem-estar, tanto dos idosos como cuidadores, com o objetivo de contribuírem para regulação de emoçóes, estresse e manutenção do ambiente familiar saudável e sem violência. ${ }^{(26,27,30)}$ Portanto, cabe aos profissionais de saúde a conscientização dos familiares acerca dos riscos do consumo de substâncias e das situações que tornam o idoso vulnerável à violência autoinfligida.

Apesar da necessidade de isolamento, o atendimento psicoterapêutico online foi recurso difundido na pandemia, e pode ser estratégia alternativa para a família e para o idoso. Quanto às atividades artísticas, estas são importantes para a cognição e promoção da saúde mental, a pessoa idosa pode ser estimulada, por exemplo, ao assistir filmes, transmissóes online de peças de teatro e musicais, praticar pintura, artes plásticas, costura, canto, leitura, entre outras.

As limitações na prestação de cuidados ao idoso de modo presencial, pode levar ao aumento da sobrecarga familiar e propensão a abusos e negligência. Além disso, tem-se o distanciamento social, que dificulta a possibilidade de uma testemunha presenciar comportamentos potencialmente abusivos ou 
negligentes com a pessoa idosa. ${ }^{(19,24,28)}$ Assim, muitos cuidadores optaram pelo uso das tecnologias virtuais para avaliação e monitoramento. Porém, alguns idosos podem não ter habilidade necessária ou apresentarem deficiência física ou mental para utilizar essas ferramentas. O uso torna-se ainda mais desafiador pela possibilidade de o abusador dividir o mesmo ambiente com o idoso e o intimidar ou impedir acesso a essa tecnologia. ${ }^{(19,24)}$ Logo, uma estratégia potencial para abordar idosos em risco é realizar ligaçóes sem aviso prévio ou agendamento. ${ }^{(19,24,25)}$

No tocante as ILPI, a suspensão ou redução de visitas e o aumento do absenteísmo da equipe podem aumentar o risco de abuso e negligência, além dos sentimentos de solidão, abandono e desânimo dos idosos. Assim, há necessidade de intensificação das inspeções, contratação de profissionais substitutos daqueles afastados, avaliação da negligência durante a pandemia e divulgação dos mecanismos de denúncia da ILPI. ${ }^{(21,22)}$ Nesse momento, é prudente a atuação dos profissionais da assistência social, que podem intervir, a partir de procedimentos legais, junto essas instituiçóes, a fim de garantir a proteção ao idoso.

Entre as açóes de enfrentamento da violência contra os idosos, aponta-se a relevância dos serviços comunitários como delegacias, conselhos, associações e abrigos. Ademais, é significativo o apoio de organizaçôes religiosas e sem fins lucrativos, vizinhos, porteiros e pessoas da comunidade para identificação de idosos em risco. ${ }^{(19,25,28)}$

Nota-se, assim, que a dificuldade de identificação dos casos de violência contra idosos exige mobilização sistêmica de diferentes setores da sociedade e parcerias institucionais que incentivem as denúncias. Ainda que mecanismos tecnológicos tenham sido desenvolvidos ou aprimorados na pandemia, tais como boletim de ocorrência online, acesso virtual a cultos e a representantes religiosos, ainda permanece o desafio da limitaçáo do acesso a esses instrumentos. Dessa forma, são necessárias discussões minuciosas, entre essas instituiçôes, sobre alternativas que visem fortalecer as conexóes da pessoa idosa com a rede de apoio social.

Os cuidadores também necessitam de cuidados durante a pandemia. A criaçáo de espaços para o acolhimento de suas emoçóes, o acompanhamento psicossocial e o estímulo para realização de atividades de lazer podem contribuir para prevenção de desgastes emocionais. Espaços para acolhimento aos cuidadores podem ser iniciativas de profissionais da atenção primária à saúde, de associações e conselhos municipais, universidades, instituiçôes religiosas ou líderes comunitários. Grupos virtuais de apoio coletivo podem ser formados, para compartilhamento de experiências e orientaçóes.

O compartilhamento do cuidado ao idoso entre pessoas que residem no domicílio deve ser considerado, de modo que a família também se sinta responsável pelos cuidados ao idoso e ao cuidador. Essa iniciativa pode ser estimulada pelo enfermeiro da atençáo primária à saúde, que pode orientar o cuidado. A implementação dessas açôes contribui para promoção da saúde das pessoas envolvidas na rede de cuidados à pessoa idosa.

Para prevenção das violências deve-se considerar o envolvimento de políticas públicas de saúde, assistência social, comunidade, família, economia, segurança e da justiça em açóes de proteção de direitos e notificação, além de cuidados aos idosos violentados. ${ }^{(30)}$ Entretanto, a escassez de políticas públicas e sociais, voltadas para açóes de enfrentamento às vulnerabilidades desse público na pandemia, sustenta o cenário complexo frente a necessidade de prevenção, identificação e responsabilização da violência contra a pessoa idosa.

As equipes multidisciplinares de saúde podem atuar na promoção de abordagens preventivas na comunidade, por meio de campanhas informativas para o público geral e idosos. É premente a atuação do enfermeiro na conscientização de sinais de abuso, incentivo ao autocuidado e práticas de bem-estar, capacitação dos idosos, desde alertar sobre golpes financeiros até instruçôes sobre saúde, recomendaçóes de higiene e gerenciamento do estresse. $(19,20)$ Outrossim, é essencial o papel do enfermeiro diante das crises humanitárias, haja vista a necessidade de cuidado numa abordagem holística. ${ }^{(32)}$ Os enfermeiros devem, portanto, reconhecer o seu papel ético, enquanto profissão e membros da sociedade civil e atuar, mesmo em meio às restriçóes ocasionadas pela pandemia COVID-19, de forma 
segura, na prevenção da violência contra a pessoa idosa em todos os espaços inerentes à sua atuação.

Apontam-se como limitaçóes desta pesquisa a ausência de estudos epidemiológicos que evidenciassem a incidência e a prevalência da violência contra a pessoa idosa. Acredita-se que isto deve-se ao fato da complexidade na condução de pesquisas que envolvem esse objeto de estudo, sobretudo ao considerar o cenário pandêmico e devido a subnotificaçáo associada ao isolamento social. Ressalta-se ainda a ausência de estudos primários sobre a COVID-19, o que pode estar relacionado ao fato de tratar-se de temática emergente, e que os estudos recentes sobre a doença têm priorizado temas como tratamento, imunização e prevenção. Ademais, verificou-se que nenhum artigo aprofundou, de forma específica, a discussão a respeito dos tipos de violências contra idosos praticados durante a pandemia, de forma que limitou o detalhamento dessa tipologia.

Este estudo apresenta potencial para fomentar e subsidiar novas investigaçóes por meio da realização de estudos originais, que auxiliem na prática do combate à violência contra a pessoa idosa no contexto da pandemia COVID-19, bem como para outras pandemias ou problemas de saúde que adotem o isolamento social como medida preventiva.

\section{Conclusão}

Estudos da violência contra idosos durante a pandemia COVID-19 ainda são escassos, de forma que a carência de evidências torna inviável a condução de uma revisão sistemática. O tema é emergente e os artigos identificados refletiram acerca de questôes sociais, institucionais e políticas na pandemia. No entanto, não abordaram, de forma específica, os tipos de violência. Ademais, permitiram a compreensão dos riscos do fenômeno na comunidade e nas ILPI durante a pandemia COVID-19, assim como condiçôes que podem agravar esses riscos e açôes políticas, organizacionais, profissionais e sociais para enfrentamento da violência, e açôes necessárias nas ILPI. O isolamento social, apesar de ser necessário para controle do contágio da doença, apresentou-se como principal fator de risco, que agrava e tornam complexas as medidas necessárias para prevenção, identificação e responsabilização da violência. Existe lacuna nas evidências de estudos empíricos sobre o tema, que apresentem dados epidemiológicos e clínicos da violência na pandemia COVID-19, bem como relativos à vulnerabilidade social dos idosos, que se torna agravada diante da crise sanitária. Ao considerar que a produção do conhecimento sobre o tema encontra-se em desenvolvimento, é imperativa a atualização e continuidade desta revisão.

\section{Referências}

1. World Health Organization (WHO). Timeline of WHO's response to COVID-19. Geneva: WHO; 2020 [cited 2020 Sep 25]. Available from: https://www.who.int/news-room/detail/29-06-2020-covidtimeline

2. World Health Organization (WHO). Director-General's opening remarks at the media briefing on COVID-19 - 11 March 2020. Genève: WHO; 2020 [cited 2020 Sep 25]. Available from: https://www.who.int/dg/ speeches/detail/who-director-general-s-opening-remarks-at-themedia-briefing-on-covid-19---11-march-2020

3. Li G, De Clercq E. Therapeutic options for the 2019 novel coronavirus (2019-nCoV). Nat Rev Drug Discov. 2020;19(3):149-50.

4. World Health Organization (WHO). COVID-19 weekly epidemiological update. Genève: WHO; 2021 [cited 2021 Mar 15]. Available from: $\quad$ https://www.who.int/docs/default-source/coronaviruse/ situation-reports/20210309_weekly_epi_update_30. pdf?sfvrsn=4e7da248_8\&download=true

5. The Center for Evidence-Based Medicine (CEBM). Evidence Service to support the COVID-19 response. Global Covid-19 Case Fatality Rates. Oxford: Oxford COVID Evidence Service; 2020 [cited 2020 Nov 3]. Available from: https://www.cebm.net/covid-19/global-covid-19case-fatality-rates/

6. Liu K, Chen Y, Lin R, Han K. Clinical features of COVID-19 in elderly patients: a comparison with young and middle-aged patients. Journal of Infection. J Infect. 2020;80(6):14-18.

7. Radwan E, Radwan A, Radwan W. Challenges facing older adults during the COVID-19 outbreak. European J Env Publi Health. 2021;5(1):em0059.

8. Brasil. Secretaria de Direitos Humanos da Presidência da República. Brasil: manual de enfrentamento à violência contra a pessoa idosa. É possível prevenir. É necessário superar. Brasília (DF): Secretaria de Direitos Humanos da Presidência da República; 2013 [citado 2020 Nov 3]. Disponível em: http://www.dive.sc.gov.br/conteudos/agravos/ publicacoes/manual-de-enfrentamento-a-violencia-contra-a-pessoaidosa.pdf

9. Pillemer K, Burnes D, Riffin C, Lachs MS. Elder abuse: global situation, risk factors, and prevention strategies. Gerontologist. 2016;56(Suppl 2):S194-205. Review.

10. Storey JE. Risk factors for elder abuse and neglect: a review of the literature. Aggression Violent Behavior. 2020;50:101339. 
11. World Health Organization (WHO). Addressing violence against children, women and older people during the COVID-19 pandemic: key actions. Genève: WHO; 2020 [cited 2020 Nov 3]. Available from: https://www. who.int/publications//item/WHO-2019-nCoV-Violence_actions-2020.1

12. Brasil. Governo do Brasil. Disque 100 - Aumenta número de denúncias de violação aos direitos de idosos durante pandemia. Brasília (DF): Governo do Brasil; 2020 [citado 2020 Nov 3]. Disponível em: https://www.gov. br/pt-br/noticias/assistencia-social/2020/06/aumenta-numero-dedenuncias-de-violacao-aos-direitos-de-idosos-durante-pandemia

13. Arksey H, O'Malley L. Scoping studies: towards a methodological framework. Int J Soc Res Methodol. 2005;8(1):19-32.

14. Peters M, Godfrey CM, Mcinerney P, Baldini Soares C, Khalil $H$, Parker D. Scoping reviews. In: Aromataris E, Munn Z, editors. Joanna Briggs Institute Reviewer's Manual, 2017 [cited 2020 Sep 25]. Available from: https://wiki.jbi.global/display/MANUAL/ Chapter+11\%3A+Scoping+reviews

15. Ouzzani M, Hammady H, Fedorowicz Z, Elmagarmid A. Rayyan-a web and mobile app for systematic reviews. Syst Rev. 2016;5(1):210. Review.

16. The Joanna Briggs Institute. Joanna Briggs Institute Reviewers' Manual: 2015 edition/ Supplement. Australia: The Joanna Briggs Institute; 2015 [cited Sep 26]. Available from: https://nursing.Isuhsc. edu/JBI/docs/ReviewersManuals/Scoping-.pdf

17. Whittemore R, Knafl K. The integrative review: updated methodology. J Adv Nurs. 2005; 52(5):546-53.

18. Tricco AC, Lillie E, Zarin W, O'Brien KK, Colquhoun $H$, Levac D, et al. PRISMA Extension for scoping reviews (PRISMA-ScR): checklist and explanation. Ann Intern Med. 2018;169(7):467-73.

19. Elman A, Breckmanc R, Clark S, Gottesman E, Rachmuth L, Reiff M, et al. Effects of the COVID-19 outbreak on elder mistreatment and response in New York City: Initial Lessons. J Appl Gerontol. 2020;39(7):690-9.

20. Gray V. Protecting the elderly through and beyond the Covid-19 lockdown. J Community Nurs. 2020;34(3):12-3.
21. Gardner W, States D, Bagley N. The coronavirus and the risks to the elderly in long-term care. J Aging Soc Policy. 2020;32(4-5):310-5.

22. Jolly A. Statutory neglect and care in a pandemic. Int Soc Work. 2020;63(5):671-5.

23. Platts-Mills TF, Hurka-Richardson K. Strengthening our intuition about elder abuse. Ann Emerg Med. 2020;76(3):277-9.

24. Makaroun LK, Bachrach RL, Rosland A. Elder abuse in the time of COVID-19 - increased risks for older adults and their caregivers. Am J Geriatric Psychiatry. 2020;28(8):876-80.

25. Han SD, Mosqueda L. Elder abuse in the COVID-19 era. J Am Geriatr Soc. 2020;68(7):1386-7.

26. Karimian M, Mansouri F, Gheiasi G, Solaimanizadeh L, Otaghi M, Salimi $E$, et al. Psychological abuse in the elderly during exchanging news of COVID-19. Iran J Psychiatry Behav Sci. 2020;14(2):e103932.

27. Conejero I, Berrouiguet S, Ducasse D, Leboyer M, Jardon V, Olié E, et al. Épidémie de COVID-19 et prise en charge des conduites suicidaires: challenge et perspectives. Encephale. 2020;46(3S):S66-S72. Review.

28. Greenberg NE, Wallick A, Brown LM. Impact of COVID-19 pandemic restrictions on community-dwelling caregivers and persons with dementia. Psychol Trauma. 2020;12(S1):S220-1.

29. Rina K, Maiti T, Panigrahi M, Patro B, Kar N, Padhy SK. Impact of COVID-19 pandemic and lockdown on elder abuse. J Geriatric Care Res. 2020;7(3):103-7.

30. Moraes CL, Marques ES, Ribeiro AP, Souza ER. Contributions to address violence against older adults during the Covid-19 pandemic in Brazil. Cien Saude Colet. 2020;25(Supl.2):4177-84.

31. Taha S, Matheson K, Cronin T, Anisman H. Intolerance of uncertainty, appraisals, coping, and anxiety: the case of the 2009 H1N1 pandemic. Br J Health Psychol. 2014;19(3):592-605.

32. The Lancet. 2020: unleashing the full potential of nursing [editorial]. Lancet. 2019;394(10212):1879. 